\title{
Microwave-assisted synthesis and pharmacological screening of some triazolothiadiazole derivatives
}

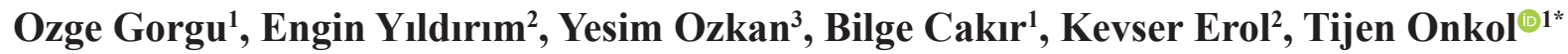 \\ ${ }^{1}$ Department of Pharmaceutical Chemistry, Faculty of Pharmacy, Gazi University, Ankara, Turkey, ${ }^{2}$ Department of \\ Pharmacology, Faculty of Medicine, Osmangazi University, Eskisehir, Turkey, ${ }^{3}$ Department of Biochemistry, Faculty of \\ Pharmacy, Gazi University, Ankara, Turkey
}

\begin{abstract}
In this study, twenty-two new $[1,2,4]$ triazolo[3,4-b][1,3,4]thiadiazoles (5a-n, 6a-h) were synthesized under microwave irradiation (MWI). The chemical structures of the compounds were elucidated by their IR, ${ }^{1} \mathrm{H}-\mathrm{NMR}, \mathrm{LC}-\mathrm{MS}$, and elemental analysis. The compounds were tested for antinociceptive activity by using the tail clip, tail flick, hot plate, and writhing methods in mice. The varying levels of antinociceptive activity of the compounds were compared with those of aspirin. Among these compounds, compound $\mathbf{5 g}$ and $\mathbf{5 j}$ were found to be significantly more active than the other compounds and the standard in the tests. Also, inhibitory effects of the test compounds on COX-1 and COX-2 activities were investigated. DuP-697 for COX-2 and SC-560 for COX-1 were used as reference standards.
\end{abstract}

Keywords: 2(3H)-Benzoxazolone. Triazolothiadiazole. Antinociceptive activity. Microwave-assisted synthesis.

\section{INTRODUCTION}

Non-steroidal anti-inflammatory drugs (NSAIDs) represent a heterogeneous family of pharmacologically active compounds used to alleviate acute and chronic inflammation, pain, and fever. Their clinical efficacy is closely related to their ability to inhibit both COX-1 and COX-2 isoforms of the enzyme cyclooxygenase (COX) which is also referred to as prostaglandin $\mathrm{H} 2$ synthase since it catalyzes the conversion of arachidonic acid to prostaglandin H2 (PGH2) (Dannhardt, Kiefer, 2001).

A large number of $\mathrm{N}$-bridged heterocycles derived from 1,2,4-thiadiazole nucleus are important pharmacological agents and there is a significant amount of research on this class of compounds. 1,2,4-Thiadiazole ring is associated with a wide variety of biological activities named antimicrobial, antimycobacterial, anticonvulsant, antidepressant, antihypertensive, and analgesic agents. Moreover, some 1,2,4-triazoles and 1,2,4-triazolo[3,4-b] $[1,3,4]$ thiadiazoles derived from 4-amino-3-thioxo-1,2,4triazoles are associated with diverse pharmacological activities such as anti-Alzheimer's (Shiradkar, et al., 2007),

\footnotetext{
*Correspondence: T. Onkol. Department of Pharmaceutical Chemistry, Faculty of Pharmacy, Gazi University, 06330, Hipodrum-Ankara-Turkey. E-mail: tijenonkol@gmail.com
}

anti-inflammatory, analgesic (Mathew, Keshavayya, Vaidya, 2006; Sar1göl et al., 2015; Haider et al., 2014; Akhter, Hassan, Amir, 2014), antiviral (Kritsanida et al., 2002), antifungal and antibacterial (Kumar et al., 2010a; Hussein et al., 2011; Aggarwal, Kumar, Dureja, 2011; Mathew et al., 2007), antitubercular (Kumar et al., 2010b; Mathew et al., 2007), and anticancer (Ibrahim, 2009) activities.

On the other hand, some compounds having small and simple $2(3 \mathrm{H})$-benzoxazolone ring show a broad spectrum of biological activity such as antimicrobial (Gülkok et al., 2012; Koksal et al., 2002), antitubercular (Gulkok et al., 2012), antioxidant (Aichaoui et al., 2009; Satyendra et al., 2011), anticonvulsant (Ucar et al., 1998), cytotoxic (Petrov et al., 2008), anti-inflammatory (Unlu et al., 2003; Dogruer et al., 1997), and analgesic (Onkol et al., 2002; Gokhan-Kelekci, Koksal, Univar, 2009; Abdelazeem et al., 2015) activities.

Design of new drugs can be based on the development of hybrid molecules by linking different pharmacophore fragments in a single structure, which may lead to compounds with interesting biological profiles.

These observations prompted us to synthesize new 1,2,4-triazolo[3,4- $b][1,3,4]$ thiadiazole derivatives, which were attached to position-3 of the $2(3 H)$-benzoxazolone ring through a methylene bridge. Also, the structure of the 
synthesized compounds was elucidated by IR, ${ }^{1} \mathrm{H}-\mathrm{NMR}$, LC-MS, and elemental analysis data.

The observed antinociceptive activities in all the tests such as hot-plate, tail-clip, tail flick and acetic acidinduced writhing tests clearly showed that mechanical, thermal and chemical nociceptive pathways had a role on their pharmacological effects (Sharma et al., 2012). Hotplate and tail-clip tests have been reported as a measure of centrally mediated transient pain (Gabra, Sirois, 2003; Wong et al., 1994).

\section{MATERIAL AND METHODS}

All chemicals and solvents used in this study were purchased from Aldrich, (Germany), Merck (Germany) and Acros (Germany) Chemical. Melting points of the compounds were recorded on an Electrothermal-9200 digital melting points apparatus and were uncorrected. Microwave reactions were carried out in MicroSYNTH Microwave Labstation at $1600 \mathrm{~W}$ (2 magnetrons $800 \mathrm{Wx} 2)$ (Milestone S.R.L. Italy). ${ }^{1} \mathrm{H}-\mathrm{NMR}$ and ${ }^{13} \mathrm{C}$ spectras of compounds were recorded in DMSO- $\mathrm{d}_{6}$ on Bruker $400 \mathrm{MHz}$ NMR spectrometer. Chemical shifts were reported in parts per million relative to internal standard tetramethylsilane. The mass spectra were recorded on a Micromass LCT Premier XE (Waters, Milford, MA, USA) LC-MS spectrometer using an positive electrospray ion source (ESI+). FTIR spectra of the surface layer of grafted membranes were measured with a Perkin-Elmer 400 (USA) ATR attachment (32 scans, wavenumber 4000-650 $\mathrm{cm}^{-1}$ ) and analyzed using the Spectrum v2.0 software. Elemental analyses were performed on Leco 932 CHNS instrument (St. Joseph, MI, USA) and were within \pm 0.4 $\%$ of the theoretical values.

\section{Chemistry}

2(3H)-Benzoxazolone (1) (Eren et al., 2010), ethyl (2(3H)-benzoxazolone-3-yl)acetate (2) (Onkol et al., 2002), (2(3H)-Benzoxazolone-3-yl)acetic acid (3) (Onkol et al. 2002), 3-[(4-amino-5-thioxo-1,2,4-triazol-3yl)methyl]-2(3H)-benzoxazolone (4) (Urlu Cicekli et al., 2012) were prepared according to the previously published procedures.

\section{General method}

General method for synthesis of 3-substituted [1,2,4] triazolo[3,4-b][1,3,4]thiadiazol-3-yl)methyl]-2(3H)benzoxazolone derivatives (5a-n) (6a-h)

To a mixture of corresponding 3-(4-pyridyl)-4- amino- 5-mercapto-1,2,4-triazole, $\mathbf{1}(0.01 \mathrm{~mol})$ and the substituted benzoic acid or substituted phenylacetic acid $(0.012 \mathrm{~mol})$ in phosphorus oxychloride $(5 \mathrm{~mL})$ were heated at $140{ }^{\circ} \mathrm{C}$ under MWI $(250 \mathrm{~W})$ for 5 to 15 minutes. The reaction mixture was slowly poured into crushed ice with stirring and neutralized with solid sodium bicarbonate. Solid product was filtered, washed with cold water, dried, and recrystallized from the appropriate solvent.

\section{Spectral data}

3-[(6-phenyl[1,2,4]triazolo[3,4-b][1,3,4]thiadiazol-3-yl) methyl]-2(3H)-benzoxazolone (5a)

Yield: $74 \%$. mp: $244-245{ }^{\circ} \mathrm{C}$. Recrystallized from EtOH-DMF. FTIR-ATR $v \max \left(\mathrm{cm}^{-1}\right): 1770(\mathrm{C}=\mathrm{O})$. ${ }^{1} \mathrm{H}-\mathrm{NMR}$ (DMSO-d $\left.6400 \mathrm{MHz}\right): \delta(\mathrm{ppm}) 5.61\left(2 \mathrm{H}, \mathrm{s}, \mathrm{CH}_{2}\right)$ ; 7.16 $\left(1 \mathrm{H}, \mathrm{td}, \mathrm{H}^{6}\right) ; 7.24\left(1 \mathrm{H}, \mathrm{td}, \mathrm{H}^{5}\right) ; 7.35-7.40(2 \mathrm{H}, \mathrm{m}$, $\left.\mathrm{H}^{4}, \mathrm{H}^{7}\right)$; 7.60-7.68 $\left(3 \mathrm{H}, \mathrm{m}\right.$, phenyl- $\left.\mathrm{H}^{3.45}\right)$; 7.87-7.89 $(2 \mathrm{H}$, $\mathrm{m}$, phenyl-H $\left.\mathrm{H}^{2.6}\right) \cdot{ }^{13} \mathrm{C}$ NMR $\left(\mathrm{DMSO}-\mathrm{d}_{6}, 100 \mathrm{MHz}\right) \delta(\mathrm{ppm})$ $167,47(\mathrm{C}=\mathrm{O}), 154.27$ (triazolothiadiazole-C), 153,95 (triazolothiadiazole-C), 142.87 (triazolothiadiazole-C), 142.44 (C), 133,51(C), 131.11 (C ), $130.20(2 \mathrm{CH})$, $129.25(\mathrm{CH}), 127.52(2 \mathrm{CH}), 124.46(\mathrm{CH}), 123.18(\mathrm{CH})$, 110.36(CH), $110.25(\mathrm{CH}), 40.81\left(\mathrm{CH}_{2}\right) . \mathrm{MS} \mathrm{ESI}(+) \mathrm{m} / \mathrm{e}$ 350.0702 (M+H, 100). Anal. calc. for $\mathrm{C}_{17} \mathrm{H}_{11} \mathrm{~N}_{5} \mathrm{O}_{2} \mathrm{~S}: \mathrm{C}$, 58.44; H, 3.17; N, 20.05; S, 9.18. Found C, 57.96; H, 3.37; N, 19.79; S, 9.30.

\section{3-\{[6-(4-fluorophenyl)[1,2,4]triazolo[3,4-b][1,3,4]}

thiadiazol-3-yl]methyl\}-2(3H)-benzoxazolone (5b)

Yield: $76 \%$. mp: $255-256{ }^{\circ} \mathrm{C}$. Recrystallized from EtOH-DMF. FTIR-ATR $v \max \left(\mathrm{cm}^{-1}\right): 1770(\mathrm{C}=\mathrm{O})$. ${ }^{1} \mathrm{H}-\mathrm{NMR}\left(\mathrm{DMSO}-\mathrm{d}_{6}\right): \delta(\mathrm{ppm}) 5.60\left(2 \mathrm{H}, \mathrm{s}, \mathrm{CH}_{2}\right), 7.16(1 \mathrm{H}$, $\left.\mathrm{t}, \mathrm{H}^{6}\right), 7.36\left(1 \mathrm{H}, \mathrm{t}, \mathrm{H}^{5}\right), 7.34-7.39\left(2 \mathrm{H}, \mathrm{m}, \mathrm{H}^{4}, \mathrm{H}^{7}\right), 7.49(2 \mathrm{H}$, $\mathrm{t}$, phenyl $\left.\mathrm{H}^{2,6}\right), 7.94-7.98\left(2 \mathrm{H}, \mathrm{m}\right.$, phenyl $\left.\mathrm{H}^{3,5}\right)$. MS ESI $(+)$ $\mathrm{m} / \mathrm{e} 368.0621(\mathrm{M}+\mathrm{H}, 100)$. Anal. calc. for $\mathrm{C}_{17} \mathrm{H}_{10} \mathrm{FN}_{5} \mathrm{O}_{2} \mathrm{~S}$ : C, 55.58; H, 2.74; N, 19.06; S, 8.73. Found C, 55.26; H, $2.91 ; \mathrm{N}, 18.67 ; \mathrm{S}, 8.78$.

\section{3-\{[6-(4-chlorophenyl)[1,2,4]triazolo[3,4-b][1,3,4]}

thiadiazol-3-yl]methyl\}-2(3H)-benzoxazolone (5c)

Yield: $73 \%$. mp: $262-263{ }^{\circ} \mathrm{C}$. Recrystallized from EtOH-DMF. FTIR-ATR $v \max \left(\mathrm{cm}^{-1}\right): 1770(\mathrm{C}=\mathrm{O})$. ${ }^{1} \mathrm{H}-\mathrm{NMR}\left(\mathrm{DMSO}-\mathrm{d}_{6}\right): \delta(\mathrm{ppm}) 5.60\left(2 \mathrm{H}, \mathrm{s}, \mathrm{CH}_{2}\right), 7.16$ $\left(1 \mathrm{H}, \mathrm{t}, \mathrm{H}^{6}\right), 7.37\left(1 \mathrm{H}, \mathrm{t}, \mathrm{H}^{5}\right), 7.35-7.39\left(2 \mathrm{H}, \mathrm{m}, \mathrm{H}^{4}, \mathrm{H}^{7}\right), 7.71$ $\left(2 \mathrm{H}, \mathrm{d}\right.$, phenyl $\left.\mathrm{H}^{2,6}\right), 7.90\left(2 \mathrm{H}, \mathrm{d}\right.$, phenyl $\left.\mathrm{H}^{3,5}\right)$. MS ESI $(+)$ m/e 384.0329 (M+H, 100). Anal. calc. for $\mathrm{C}_{17} \mathrm{H}_{10} \mathrm{ClN}_{5} \mathrm{O}_{2} \mathrm{~S}$ : C, 53.20; H, 2.63; N, 18.25; S, 8.35. Found C, 52.92; H, $2.81 ; \mathrm{N}, 18.03 ; \mathrm{S}, 8.43$. 
3-\{[6-(4-bromophenyl)[1,2,4]triazolo[3,4-b][1,3,4] thiadiazol-3-yl]methyl\}-2(3H)-benzoxazolone (5d)

Yield: $72 \%$. mp: $266-267^{\circ} \mathrm{C}$. Recrystallized from EtOH-DMF. FTIR-ATR $v \max \left(\mathrm{cm}^{-1}\right): 1760(\mathrm{C}=\mathrm{O})$. ${ }^{1} \mathrm{H}-\mathrm{NMR}\left(\mathrm{DMSO}-\mathrm{d}_{6}\right): \delta(\mathrm{ppm}) 5.60\left(2 \mathrm{H}, \mathrm{s}, \mathrm{CH}_{2}\right), 7.16(1 \mathrm{H}$, $\left.\mathrm{t}, \mathrm{H}^{6}\right), 7.24\left(1 \mathrm{H}, \mathrm{t}, \mathrm{H}^{5}\right), 7.35-7.39\left(2 \mathrm{H}, \mathrm{m}, \mathrm{H}^{4}, \mathrm{H}^{7}\right), 7.81-$ 7.86 (4H, m, phenyl H). MS ESI $(+) \mathrm{m} / \mathrm{e} 427.9806(\mathrm{M}+\mathrm{H}$, 100). Anal. calc. for $\mathrm{C}_{17} \mathrm{H}_{10} \mathrm{BrN}_{5} \mathrm{O}_{2} \mathrm{~S}: \mathrm{C}, 47.68 ; \mathrm{H}, 2.35 ; \mathrm{N}$, $16.35 ;$ S, 7.49. Found C, 47.50; H, 2.53; N, 16.20; S, 7.56.

3-\{[6-(4-methoxyphenyl)[1,2,4]triazolo[3,4-b][1,3,4] thiadiazol-3-yl]methyl\}-2(3H)-benzoxazolone (5e)

Yield: $67 \%$. mp: $235-236^{\circ} \mathrm{C}$. Recrystallized from EtOH-DMF. FTIR-ATR $v \max \left(\mathrm{cm}^{-1}\right): 1770(\mathrm{C}=\mathrm{O})$. ${ }^{1} \mathrm{H}-\mathrm{NMR}\left(\mathrm{DMSO}-\mathrm{d}_{6}\right): \delta(\mathrm{ppm}) 3.83\left(3 \mathrm{H}, \mathrm{s}, \mathrm{CH}_{3}\right), 5.55(2 \mathrm{H}$, $\left.\mathrm{s}, \mathrm{CH}_{2}\right), 7.11-7.15\left(3 \mathrm{H}, \mathrm{m}, \mathrm{H}^{6}\right.$, phenyl $\left.\mathrm{H}^{2,6}\right), 7.20(1 \mathrm{H}$, td, $\left.\mathrm{H}^{5}\right)$, 7.30-7.36 (2H, m, $\left.\mathrm{H}^{4}, \mathrm{H}^{7}\right), 7.79\left(2 \mathrm{H}\right.$, d, phenyl $\left.\mathrm{H}^{3,5}\right)$. MS ESI $(+)$ m/e $380.0742(\mathrm{M}+\mathrm{H}, 100)$. Anal. calc. for $\mathrm{C}_{18} \mathrm{H}_{13} \mathrm{~N}_{5} \mathrm{O}_{3} \mathrm{~S}: \mathrm{C}, 56.98 ; \mathrm{H}, 3.45 ; \mathrm{N}, 18.46 ; \mathrm{S}, 8.45$. Found C, 57.08; H, 3.50; N, 18.32; S, 8.56.

3-\{[6-(4-tert-butylphenyl)[1,2,4]triazolo[3,4-b][1,3,4] thiadiazol-3-yl]methyl\}-2(3H)-benzoxazolone (5f)

Yield: 59\%. mp: $197-198^{\circ} \mathrm{C}$. Recrystallized from EtOH-DMF. FTIR-ATR $v \max \left(\mathrm{cm}^{-1}\right): 1768(\mathrm{C}=\mathrm{O})$. ${ }^{1} \mathrm{H}-\mathrm{NMR}\left(\mathrm{DMSO}-\mathrm{d}_{6}\right): \delta(\mathrm{ppm}) 1.32\left(9 \mathrm{H}, \mathrm{s}, \mathrm{CH}_{3}\right), 5.60(2 \mathrm{H}$, s, $\left.\mathrm{CH}_{2}\right), 7.16\left(1 \mathrm{H}, \mathrm{td}, \mathrm{H}^{6}\right), 7.24\left(1 \mathrm{H}, \mathrm{td}, \mathrm{H}^{5}\right), 7.34-7.40(2 \mathrm{H}$, $\left.\mathrm{m}, \mathrm{H}^{4}, \mathrm{H}^{7}\right), 7.64\left(2 \mathrm{H}, \mathrm{d}\right.$, phenyl $\left.\mathrm{H}^{2,6}\right), 7.80(2 \mathrm{H}, \mathrm{d}$, phenyl $\left.\mathrm{H}^{3,5}\right)$. MS ESI $(+) \mathrm{m} / \mathrm{e} 406.1354(\mathrm{M}+\mathrm{H}, 100)$. Anal. calc. for $\mathrm{C}_{21} \mathrm{H}_{19} \mathrm{~N}_{5} \mathrm{O}_{2} \mathrm{~S}: \mathrm{C}, 62.21 ; \mathrm{H}, 4.72 ; \mathrm{N}, 17.27 ; \mathrm{S}, 7.91$. Found C, 62.39; H, 4.70; N, 17.21; S, 8.07.

3-\{[6-(4-methylphenyl)[1,2,4]triazolo[3,4-b][1,3,4] thiadiazol-3-yl]methyl\}-2(3H)-benzoxazolone (5g)

Yield: $60 \%$. mp: $247-248{ }^{\circ} \mathrm{C}$. Recrystallized from DMF- $\mathrm{H}_{2} \mathrm{O}$. FTIR-ATR $v \max \left(\mathrm{cm}^{-1}\right): 1770(\mathrm{C}=\mathrm{O})$. ${ }^{1} \mathrm{H}-\mathrm{NMR}\left(\mathrm{DMSO}-\mathrm{d}_{6}\right): \delta(\mathrm{ppm}) 2.40\left(3 \mathrm{H}, \mathrm{s}, \mathrm{CH}_{3}\right), 5.59(2 \mathrm{H}$, s, $\left.\mathrm{CH}_{2}\right), 7.16\left(1 \mathrm{H}, \mathrm{td}, \mathrm{H}^{6}\right), 7.23\left(1 \mathrm{H}, \mathrm{td}, \mathrm{H}^{5}\right), 7.35-7.39(2 \mathrm{H}$, $\left.\mathrm{m}, \mathrm{H}^{4}, \mathrm{H}^{7}\right), 7.43\left(2 \mathrm{H}, \mathrm{d}\right.$, phenyl $\left.\mathrm{H}^{2,6}\right), 7.77(2 \mathrm{H}, \mathrm{d}$, phenyl $\mathrm{H}$ $3,5)$. MS ESI $(+) \mathrm{m} / \mathrm{e} 364.0858(\mathrm{M}+\mathrm{H}, 100)$. Anal. calc. for $\mathrm{C}_{18} \mathrm{H}_{13} \mathrm{~N}_{5} \mathrm{O}_{2} \mathrm{~S}: \mathrm{C}, 59.49 ; \mathrm{H}, 3.61 ; \mathrm{N}, 19.27 ; \mathrm{S}, 8.82$. Found C, 59.44;H, 3.80; N, 19.04; S, 8.97.

3-(\{6-[4-(trifluoromethyl)phenyl][1,2,4]triazolo[3,4-b] [1,3,4]thiadiazol-3-yl\}methyl)-2(3H)-benzoxazolone (5h)

Yield: $67 \%$. mp: $307-308{ }^{\circ} \mathrm{C}$. Recrystallized from DMF-H ${ }_{2} \mathrm{O}$. FTIR-ATR $v \max \left(\mathrm{cm}^{-1}\right): 1769(\mathrm{C}=\mathrm{O})$. ${ }^{1} \mathrm{H}-\mathrm{NMR}\left(\mathrm{DMSO}-\mathrm{d}_{6}\right): \delta(\mathrm{ppm}) 5.63\left(2 \mathrm{H}, \mathrm{s}, \mathrm{CH}_{2}\right), 7.17$ $\left(1 \mathrm{H}, \mathrm{t}, \mathrm{H}^{6}\right), 7.25\left(1 \mathrm{H}, \mathrm{t}, \mathrm{H}^{5}\right), 7.36-7.40\left(2 \mathrm{H}, \mathrm{m}, \mathrm{H}^{4}, \mathrm{H}^{7}\right), 8.01$ $\left(2 \mathrm{H}, \mathrm{d}\right.$, phenyl $\left.\mathrm{H}^{2,6}\right), 8.11\left(2 \mathrm{H}, \mathrm{d}\right.$, phenyl $\left.\mathrm{H}^{3,5}\right)$. MS ESI $(+)$ $\mathrm{m} / \mathrm{e} 418.0584(\mathrm{M}+\mathrm{H}, 100)$. Anal. calc. for $\mathrm{C}_{18} \mathrm{H}_{10} \mathrm{~F}_{3} \mathrm{~N}_{5} \mathrm{O}_{2} \mathrm{~S}$ :
C,. 51.80; H, 2.42; N, 16.78; S, 7.68. Found C, 51.55; H, $2.59 ; \mathrm{N}, 16.65 ; \mathrm{S}, 7.78$.

3-(\{6-[4-(methylsulfonyl)phenyl][1,2,4]triazolo[3,4-b] [1,3,4]thiadiazol-3-yl\}methyl)-2(3H)-benzoxazolone (5i) Yield: $70 \%$. mp: $302-303{ }^{\circ} \mathrm{C}$. Recrystallized from DMF- $\mathrm{H}_{2} \mathrm{O}$. FTIR-ATR $v \max \left(\mathrm{cm}^{-1}\right): 1770(\mathrm{C}=\mathrm{O})$. ${ }^{1} \mathrm{H}-\mathrm{NMR}\left(\mathrm{DMSO}_{\mathrm{d}}\right)$ : $\delta(\mathrm{ppm}) 3.32\left(3 \mathrm{H}, \mathrm{s}, \mathrm{SO}_{2} \mathrm{CH}_{3}\right), 5.63$ $\left(2 \mathrm{H}, \mathrm{s}, \mathrm{CH}_{2}\right), 7.17\left(1 \mathrm{H}, \mathrm{t}, \mathrm{H}^{6}\right), 7.26\left(1 \mathrm{H}, \mathrm{t}, \mathrm{H}^{5}\right), 7.38-7.41$ $\left(2 \mathrm{H}, \mathrm{m}, \mathrm{H}^{4}, \mathrm{H}^{7}\right), 8.13-8.18(4 \mathrm{H}, \mathrm{m}$, phenyl H). MS ESI $(+)$ $\mathrm{m} / \mathrm{e} 428.0490(\mathrm{M}+\mathrm{H}, 100)$. Anal. calc. for $\mathrm{C}_{18} \mathrm{H}_{13} \mathrm{~N}_{5} \mathrm{O}_{4} \mathrm{~S}_{2}$ : C, 50.58; H, 3.07; N, 16.38; S, 15.00. Found C, 50.91; H, $3.18 ; \mathrm{N}, 16.41 ; \mathrm{S}, 14.97$.

\section{3-\{[6-(4-nitrophenyl)[1,2,4]triazolo[3,4-b][1,3,4]} thiadiazol-3-yl]methyl\}-2(3H)-benzoxazolone (5j)

Yield: $66 \%$. mp: $283-284{ }^{\circ} \mathrm{C}$. Recrystallized from EtOH-DMF. FTIR-ATR $v \max \left(\mathrm{cm}^{-1}\right): 1769(\mathrm{C}=\mathrm{O})$. ${ }^{1} \mathrm{H}-\mathrm{NMR}\left(\mathrm{DMSO}-\mathrm{d}_{6}\right): \delta(\mathrm{ppm}) 5.63\left(2 \mathrm{H}, \mathrm{s}, \mathrm{CH}_{2}\right), 7.17$ $\left(1 \mathrm{H}, \mathrm{t}, \mathrm{H}^{6}\right), 7.26\left(1 \mathrm{H}, \mathrm{t}, \mathrm{H}^{5}\right), 7.37-7.40\left(2 \mathrm{H}, \mathrm{m}, \mathrm{H}^{4}, \mathrm{H}^{7}\right), 8.16$ $\left(2 \mathrm{H}, \mathrm{d}\right.$, phenyl- $\left.\mathrm{H}^{2,6}\right), 8.45\left(2 \mathrm{H}, \mathrm{d}\right.$, phenyl- $\left.\mathrm{H}^{3,5}\right)$. MS ESI $(+)$ m/e $395.0558(\mathrm{M}+\mathrm{H}, 100)$. Anal. calc. for $\mathrm{C}_{17} \mathrm{H}_{10} \mathrm{~N}_{6} \mathrm{O}_{4} \mathrm{~S}$ : C, 51.77; H, 2.56; N, 21.31; S, 8.13. Found C, 52.13; H, $2.69 ; \mathrm{N}, 21.31 ; \mathrm{S}, 8.31$.

\section{3-\{[6-(4-cyanophenyl)[1,2,4]triazolo[3,4-b][1,3,4]} thiadiazol-3-yl]methyl\}-2(3H)-benzoxazolone (5k)

Yield: $70 \%$. mp: $272-273{ }^{\circ} \mathrm{C}$. Recrystallized from EtOH-DMF. FTIR-ATR $v \max \left(\mathrm{cm}^{-1}\right): 1770(\mathrm{C}=\mathrm{O})$. ${ }^{1} \mathrm{H}-\mathrm{NMR}\left(\mathrm{DMSO}-\mathrm{d}_{6}\right): \delta(\mathrm{ppm}) 5.62\left(2 \mathrm{H}, \mathrm{s}, \mathrm{CH}_{2}\right), 7.16(1 \mathrm{H}$, td, $\left.\mathrm{H}^{6}\right), 7.24\left(1 \mathrm{H}, \mathrm{t}, \mathrm{H}^{5}\right), 7.35-7.39\left(2 \mathrm{H}, \mathrm{m}, \mathrm{H}^{4}, \mathrm{H}^{7}\right), 8.05-$ $8.12(4 \mathrm{H}, \mathrm{m}$, phenyl-H). MS ESI $(+) \mathrm{m} / \mathrm{e} 375.0659(\mathrm{M}+\mathrm{H}$, 100). Anal. calc. for $\mathrm{C}_{18} \mathrm{H}_{10} \mathrm{~N}_{6} \mathrm{O}_{2} \mathrm{~S}: \mathrm{C}, 57.75 ; \mathrm{H}, 2.69 ; \mathrm{N}$, $22.45 ; \mathrm{S}, 8.56$. Found C, 57.67; H, 2.79; N, 22.18; S, 8.66.

\section{3-\{[6-(pyridin-4-yl)[1,2,4]triazolo[3,4-b][1,3,4]}

thiadiazol-3-yl]methyl\}-2(3H)-benzoxazolone (5I)

Yield: $34 \%$. mp: $282-283{ }^{\circ} \mathrm{C}$. Recrystallized from EtOH-DMF. FTIR-ATR $v \max \left(\mathrm{cm}^{-1}\right): 1757(\mathrm{C}=\mathrm{O})$. ${ }^{1} \mathrm{H}-\mathrm{NMR}\left(\mathrm{DMSO}-\mathrm{d}_{6}\right): \delta(\mathrm{ppm}) 5.63\left(2 \mathrm{H}, \mathrm{s}, \mathrm{CH}_{2}\right), 7.17(1 \mathrm{H}$, $\left.\mathrm{t}, \mathrm{H}^{6}\right), 7.25\left(1 \mathrm{H}, \mathrm{t}, \mathrm{H}^{5}\right), 7.36-7.40\left(2 \mathrm{H}, \mathrm{m}, \mathrm{H}^{4}, \mathrm{H}^{7}\right), 7.84(2 \mathrm{H}$, $\mathrm{d}$, pyridin $\left.\mathrm{H}^{2,6}\right), 8.85\left(2 \mathrm{H}, \mathrm{d}\right.$, pyridin $\left.\mathrm{H}^{3,5}\right)$. MS ESI $(+) \mathrm{m} / \mathrm{e}$ $351.0664(\mathrm{M}+\mathrm{H}, 100)$. Anal. calc. for $\left.\mathrm{C}_{16} \mathrm{H}_{10} \mathrm{~N}_{6} \mathrm{O}_{2} \mathrm{~S}\right)$ : C, $\mathrm{H}, \mathrm{N}, \mathrm{S}$ calc. 54.85, 2.88, 23.99, 9.15 found 54.43, 3.01, $23.59,9.17$.

3-\{[6-(thiophen-3-yl)[1,2,4]triazolo[3,4-b][1,3,4] thiadiazol-3-yl]methyl\}-2(3H)-benzoxazolone (5m)

Yield: $50 \%$. mp: $233-234{ }^{\circ} \mathrm{C}$. Recrystallized from EtOH-DMF. FTIR-ATR $v \max \left(\mathrm{cm}^{-1}\right): 1769(\mathrm{C}=\mathrm{O})$. ${ }^{1} \mathrm{H}-\mathrm{NMR}\left(\mathrm{DMSO}-\mathrm{d}_{6}\right): \delta(\mathrm{ppm}) 5,58\left(2 \mathrm{H}, \mathrm{s}, \mathrm{CH}_{2}\right) ; 7,16(1 \mathrm{H}$ 
, td , $\left.\mathrm{H}^{6}\right) ; 7,24\left(1 \mathrm{H}, \mathrm{td}, \mathrm{H}^{5}\right) ; 7,35-7,40\left(2 \mathrm{H}, \mathrm{m}, \mathrm{H}^{7}, 4\right)$ ; 7,51-7,52 $\left(1 \mathrm{H}, \mathrm{m}\right.$, thienyl- $\left.\mathrm{H}^{4}\right) ; 7,84-7,86(1 \mathrm{H}, \mathrm{m}$, thienyl- $\left.\mathrm{H}^{5}\right) ; 8,47-8,48\left(1 \mathrm{H}, \mathrm{m}\right.$, thienyl- $\left.\mathrm{H}^{2}\right)$. MS ESI $(+)$ $\mathrm{m} / \mathrm{e} 356.0278(\mathrm{M}+\mathrm{H}, 100)$. Anal. calc. for $\mathrm{C}_{15} \mathrm{H}_{9} \mathrm{~N}_{5} \mathrm{O}_{2} \mathrm{~S}_{2}$ : C, 50.69; H, 2.55; N, 19.71; S, 18.04. Found C, 53.04; H, $2.81 ; \mathrm{N}, 20.43 ; \mathrm{S}, 18.12$.

3-\{[6-(furan-3-yl)[1,2,4]triazolo[3,4-b][ 1,3,4]thiadiazol3-yl]methyl\}-2(3H)-benzoxazolone (5n)

Yield: $85 \%$. mp: 202-203 ${ }^{\circ} \mathrm{C}$. Recrystallized from EtOH-DMF. FTIR-ATR $v \max \left(\mathrm{cm}^{-1}\right): 1771(\mathrm{C}=\mathrm{O})$. ${ }^{1} \mathrm{H}-\mathrm{NMR}\left(\mathrm{DMSO}-\mathrm{d}_{6}\right): \delta(\mathrm{ppm}) 5,56\left(2 \mathrm{H}, \mathrm{s}, \mathrm{CH}_{2}\right) ; 6,91(1 \mathrm{H}$ , d , furan- $\left.\mathrm{H}^{4}\right) ; 7,16\left(1 \mathrm{H}, \mathrm{t}, \mathrm{H}^{6}\right) ; 7,24\left(1 \mathrm{H}, \mathrm{t}, \mathrm{H}^{5}\right) ; 7,34-$ $7,40\left(2 \mathrm{H}, \mathrm{m}, \mathrm{H}^{7,4}\right) ; 7,98-7,99\left(1 \mathrm{H}, \mathrm{m}\right.$, furan- $\left.\mathrm{H}^{5}\right) ; 8,72(1 \mathrm{H}$ , $\mathrm{m}$,furan- $\left.\mathrm{H}^{2}\right)$. MS ESI $(+) \mathrm{m} / \mathrm{e} 340.0508(\mathrm{M}+\mathrm{H}, 100)$. Anal. calc. for $\mathrm{C}_{15} \mathrm{H}_{9} \mathrm{~N}_{5} \mathrm{O}_{3} \mathrm{~S}: \mathrm{C}, 53.09 ; \mathrm{H}, 2.67 ; \mathrm{N}, 20.64$; S, 9.45. Found C, 50.94; H, 2.65; N, 19.63; S, 9.54.

3-[(6-benzyl[1,2,4]triazolo[3,4-b][1,3,4]thiadiazol-3-yl) methyl]-2(3H)-benzoxazolone (6a)

Yield: $49 \%$. mp: $188-189^{\circ} \mathrm{C}$. Recrystallized from EtOH. FTIR-ATR $v$ max $\left(\mathrm{cm}^{-1}\right): 1767(\mathrm{C}=\mathrm{O}) .{ }^{1} \mathrm{H}-\mathrm{NMR}$ $\left(\mathrm{DMSO}_{6}\right): \delta(\mathrm{ppm}) 4.42\left(2 \mathrm{H}, \mathrm{s}, \mathrm{CH}_{2}\right), 5.53\left(2 \mathrm{H}, \mathrm{s}, \mathrm{CH}_{2}\right)$, 7.22-7.14 (2H, m, $\left.\mathrm{H}^{5}, \mathrm{H}^{6}\right), 7.27-7.25\left(1 \mathrm{H}, \mathrm{m}, \mathrm{H}^{4}\right), 7.40-$ $7.31\left(6 \mathrm{H}, \mathrm{m}, \mathrm{H}^{7}\right.$, phenyl-H). MS ESI(+) m/e 364.0871 $(\mathrm{M}+\mathrm{H}, 100)$. Anal. calc. for $\mathrm{C}_{18} \mathrm{H}_{13} \mathrm{~N}_{5} \mathrm{O}_{2} \mathrm{~S}: \mathrm{C}, 59.49 ; \mathrm{H}$, $3.61 ; \mathrm{N}, 19.27 ; \mathrm{S}, 8.82$. Found C, 58.83; H, 3.79; N, 18.27; $\mathrm{S}, 8.53$.

\section{3-\{[6-(4-fluorobenzyl)[1,2,4]triazolo[3,4-b][1,3,4]} thiadiazol-3-yl]methyl\}-2(3H)-benzoxazolone (6b)

Yield: $74 \%$. mp: $194-195{ }^{\circ} \mathrm{C}$. Recrystallized from EtOH-DMF. FTIR-ATR $v \max \left(\mathrm{cm}^{-1}\right): 1765(\mathrm{C}=\mathrm{O})$. ${ }^{1} \mathrm{H}-\mathrm{NMR}\left(\mathrm{DMSO}-\mathrm{d}_{6}\right): \delta(\mathrm{ppm}) 4.39\left(2 \mathrm{H}, \mathrm{s}, \mathrm{CH}_{2}\right), 5.48(2 \mathrm{H}$, $\left.\mathrm{s}, \mathrm{CH}_{2}\right), 7.22-7.10\left(5 \mathrm{H}, \mathrm{m}, \mathrm{H}^{4}, \mathrm{H}^{5}, \mathrm{H}^{6}\right.$, phenyl- $\left.\mathrm{H}^{2,6}\right)$, 7.37$7.33\left(3 \mathrm{H}, \mathrm{m}, \mathrm{H}^{7}\right.$, phenyl- $\left.{ }^{3,5}\right)$. MS ESI(+) m/e 382.0782 $(\mathrm{M}+\mathrm{H}, 100)$. Anal. calc. for $\mathrm{C}_{18} \mathrm{H}_{12} \mathrm{FN}_{5} \mathrm{O}_{2} \mathrm{~S}: \mathrm{C}, 56.69 ; \mathrm{H}$, 3.17 ; N, 18.36; S, 8.41. Found C, 56.69; H, 3.18; N, 18.12; S, 8.45 .

\section{3-\{[6-(4-chlorobenzyl)[1,2,4]triazolo[3,4-b][1,3,4]} thiadiazol-3-yl]methyl\}-2(3H)-benzoxazolone (6c)

Yield: $72 \%$. mp: $198-199^{\circ} \mathrm{C}$. Recrystallized from EtOH-DMF. FTIR-ATR $v \max \left(\mathrm{cm}^{-1}\right): 1763(\mathrm{C}=\mathrm{O})$. ${ }^{1} \mathrm{H}-\mathrm{NMR}\left(\mathrm{DMSO}_{\mathrm{d}}\right): \delta(\mathrm{ppm}) 4.44\left(2 \mathrm{H}, \mathrm{s}, \mathrm{CH}_{2}\right), 5.52$ $\left(2 \mathrm{H}, \mathrm{s}, \mathrm{CH}_{2}\right), 7.26-7.14\left(2 \mathrm{H}, \mathrm{m}, \mathrm{H}^{5}, \mathrm{H}^{6}\right), 7.26-7.24(1 \mathrm{H}$, $\left.\mathrm{m}, \mathrm{H}^{4}\right)$, 7.44-7.36 (5H, m, $\mathrm{H}^{7}$, phenyl-H). MS ESI $(+) \mathrm{m} / \mathrm{e}$ 398.0481 (M+H, 100). Anal. calc. for $\mathrm{C}_{18} \mathrm{H}_{12} \mathrm{ClN}_{5} \mathrm{O}_{2} \mathrm{~S}: \mathrm{C}$, 54.34; H, 3.04; N, 17.60; S, 8.06. Found C, 54.22; H, 3.14; $\mathrm{N}, 17.50 ; \mathrm{S}, 8.02$. 3-\{[6-(4-methylbenzyl)[1,2,4]triazolo[3,4-b][1,3,4] thiadiazol-3-yl]methyl\}-2(3H)-benzoxazolone (6d)

Yield: $71 \%$. mp: $178-179^{\circ} \mathrm{C}$. Recrystallized from EtOH-DMF. FTIR-ATR $v \max \left(\mathrm{cm}^{-1}\right): 1767(\mathrm{C}=\mathrm{O})$. ${ }^{1} \mathrm{H}-\mathrm{NMR}\left(\mathrm{DMSO}-\mathrm{d}_{6}\right): \delta(\mathrm{ppm}) 2.29\left(3 \mathrm{H}, \mathrm{s}, \mathrm{CH}_{3}\right), 4.36$ $\left(2 \mathrm{H}, \mathrm{s}, \mathrm{CH}_{2}\right)$, ) $5.52\left(2 \mathrm{H}, \mathrm{s}, \mathrm{CH}_{2}\right), 7.27-7.16\left(7 \mathrm{H}, \mathrm{m}, \mathrm{H}^{4}\right.$, $\mathrm{H}^{5}, \mathrm{H}^{6}$, phenyl-H $), 7.39\left(1 \mathrm{H}, \mathrm{m}, \mathrm{H}^{7}\right)$. MS ESI $(+) \mathrm{m} / \mathrm{e}$ 378.1029 (M+H, 100). Anal. calc. for $\mathrm{C}_{19} \mathrm{H}_{15} \mathrm{~N}_{5} \mathrm{O}_{2} \mathrm{~S}: \mathrm{C}$, 60.46 ; H, 4.01; N, 18.56; S, 8.50. Found C, 60.01; H, 4.17; $\mathrm{N}, 17.48 ; \mathrm{S}, 8.02$.

3-\{[6-(4-methoxybenzyl)[1,2,4]triazolo[3,4-b][1,3,4] thiadiazol-3-yl]methyl\}-2(3H)-benzoxazolone (6e)

Yield: $65 \%$. mp: $145-146^{\circ} \mathrm{C}$. Recrystallized from EtOH. FTIR-ATR $v \max \left(\mathrm{cm}^{-1}\right): 1768(\mathrm{C}=\mathrm{O}) .{ }^{1} \mathrm{H}-\mathrm{NMR}$ $\left(\mathrm{DMSO}_{6}\right): \delta(\mathrm{ppm}) 3.75\left(3 \mathrm{H}, \mathrm{s}, \mathrm{OCH}_{3}\right), 4.34(2 \mathrm{H}, \mathrm{s}$, $\left.\mathrm{CH}_{2}\right), 5.52\left(2 \mathrm{H}, \mathrm{s}, \mathrm{CH}_{2}\right), 6.92\left(2 \mathrm{H}, \mathrm{d}\right.$, phenyl- $\left.\mathrm{H}^{2,6}\right), 7.22-$ $7.14\left(2 \mathrm{H}, \mathrm{m}, \mathrm{H}^{5}, \mathrm{H}^{6}\right), 7.27-7.25\left(3 \mathrm{H}, \mathrm{m}, \mathrm{H}^{4}\right.$, phenyl- $\left.\mathrm{H}^{3,5}\right)$, $7.39\left(1 \mathrm{H}, \mathrm{dd}, \mathrm{H}^{7}\right)$. MS ESI $(+) \mathrm{m} / \mathrm{e} 394.0979(\mathrm{M}+\mathrm{H}, 100)$. Anal. calc. for $\mathrm{C}_{19} \mathrm{H}_{15} \mathrm{~N}_{5} \mathrm{O}_{3} \mathrm{~S}: \mathrm{C}, 58.01 ; \mathrm{H}, 3.84 ; \mathrm{N}, 17.80$; S, 8.15. Found C, 57.53; H, 4.17; N, 16.70; S, 7.75.

3-(\{6-[4-(trifluoromethyl)benzyl][1,2,4]triazolo[3,4-b] [1,3,4]thiadiazol-3-yl\}methyl)-2(3H)-benzoxazolone (6f) Yield: $65 \%$. mp: $243-244{ }^{\circ} \mathrm{C}$. Recrystallized from EtOH-DMF. FTIR-ATR $v \max \left(\mathrm{cm}^{-1}\right): 1765(\mathrm{C}=\mathrm{O})$. ${ }^{1} \mathrm{H}-\mathrm{NMR}\left(\mathrm{DMSO}-\mathrm{d}_{6}\right): \delta(\mathrm{ppm}) 4.56\left(2 \mathrm{H}, \mathrm{s}, \mathrm{CH}_{2}\right), 5.52$ $\left(2 \mathrm{H}, \mathrm{s}, \mathrm{CH}_{2}\right), 7.20-7.13\left(2 \mathrm{H}, \mathrm{m}, \mathrm{H}^{5}, \mathrm{H}^{6}\right), 7.73(2 \mathrm{H}, \mathrm{d}$, phenyl- $\left.\mathrm{H}^{2,6}\right), 7.58\left(2 \mathrm{H}, \mathrm{d}\right.$, phenyl- $\left.\mathrm{H}^{3,5}\right), 7.25-7.23(1 \mathrm{H}$, $\left.\mathrm{m}, \mathrm{H}^{4}\right)$, 7.39-7.36 $\left(1 \mathrm{H}, \mathrm{m}, \mathrm{H}^{7}\right)$. MS ESI $(+) \mathrm{m} / \mathrm{e} 432.0750$ $(\mathrm{M}+\mathrm{H}, 100)$. Anal. calc. for $\left.\mathrm{C}_{19} \mathrm{H}_{12} \mathrm{~F}_{3} \mathrm{~N}_{5} \mathrm{O}_{2} \mathrm{~S}\right): \mathrm{C}, 52.90$; $\mathrm{H}, 2.80 ; \mathrm{N}, 16.23 ; \mathrm{S}, 7.43$. Found $\mathrm{C}, 52.57 ; \mathrm{H}, 2.96$; N, $16.20 ; \mathrm{S}, 7.48$.

3-\{[6-(4-tert-butylbenzyl)[1,2,4]triazolo[3,4-b][1,3,4] thiadiazol-3-yl]methyl\}-2(3H)-benzoxazolone (6g)

Yield: $66 \%$. mp: $154-155^{\circ} \mathrm{C}$. Recrystallized from EtOH. FTIR-ATR $v \max \left(\mathrm{cm}^{-1}\right): 1765(\mathrm{C}=\mathrm{O}) .{ }^{1} \mathrm{H}-\mathrm{NMR}$ $($ DMSO-d $)$ ): $\delta(\mathrm{ppm}) 1.23\left(9 \mathrm{H}, \mathrm{s}, \mathrm{CH}_{3}\right), 4.33\left(2 \mathrm{H}, \mathrm{s}, \mathrm{CH}_{2}\right)$, $5.49\left(2 \mathrm{H}, \mathrm{s}, \mathrm{CH}_{2}\right), 7.18-7.10\left(2 \mathrm{H}, \mathrm{m}, \mathrm{H}^{5}, \mathrm{H}^{6}\right), 7.24-7.21$ $\left(3, \mathrm{~m}, \mathrm{H}^{4}\right.$, phenyl $\left.\mathrm{H}^{2,6}\right)$, 7.37-7.33 $\left(3 \mathrm{H}, \mathrm{m}, \mathrm{H}^{7}\right.$, phenyl- $\left.\mathrm{H}^{3,5}\right)$. MS ESI $(+) \mathrm{m} / \mathrm{e} 420.1494(\mathrm{M}+\mathrm{H}, 100)$. Anal. calc. for $\mathrm{C}_{22} \mathrm{H}_{21} \mathrm{~N}_{5} \mathrm{O}_{2} \mathrm{~S}: \mathrm{C}, 62.99 ; \mathrm{H}, 5.05 ; \mathrm{N}, 16,69 ; \mathrm{S}, 7.64$. Found C, 62.97; H, 4.99; N, 16.53; S, 7.76.

3-(\{6-[4-(methylsulfonyl)benzyl][1,2,4]triazolo[3,4-b] [1,3,4]thiadiazol-3-yl\}methyl)-2(3H)-benzoxazolone (6h)

Yield: $63 \%$. mp: $247-248{ }^{\circ} \mathrm{C}$. Recrystallized from EtOH-DMF. FTIR-ATR $v \max \left(\mathrm{cm}^{-1}\right): 1765(\mathrm{C}=\mathrm{O})$. ${ }^{1} \mathrm{H}-\mathrm{NMR}\left(\mathrm{DMSO}-\mathrm{d}_{6}\right): \delta(\mathrm{ppm}) 3.23\left(3 \mathrm{H}, \mathrm{s}, \mathrm{SO}_{2} \mathrm{CH}_{3}\right)$, $4.58\left(2 \mathrm{H}, \mathrm{s}, \mathrm{CH}_{2}\right), 5.52\left(2 \mathrm{H}, \mathrm{s}, \mathrm{CH}_{2}\right), 7.21-7.13(2 \mathrm{H}, \mathrm{m}$, 
$\left.\mathrm{H}^{5}, \mathrm{H}^{6}\right), 7.25-7.23\left(1 \mathrm{H}, \mathrm{m}, \mathrm{H}^{4}\right), 7.39-7.37\left(1 \mathrm{H}, \mathrm{m}, \mathrm{H}^{7}\right), 7.61$ $\left(2 \mathrm{H}, \mathrm{d}\right.$, phenyl $\left.\mathrm{H}^{2,6}\right), 7.91\left(2 \mathrm{H}, \mathrm{d}\right.$, phenyl- $\left.\mathrm{H}^{3,5}\right)$. MS ESI $(+)$ $\mathrm{m} / \mathrm{e} 442.0644(\mathrm{M}+\mathrm{H}, 100)$. Anal. calc. for $\mathrm{C}_{19} \mathrm{H}_{15} \mathrm{~N}_{5} \mathrm{O}_{4} \mathrm{~S}_{2}$ : C, 51.69; H, 3.42; N, 15.86; S, 14.53. Found C, 51.76; H, $3.48 ; \mathrm{N}, 15.74 ; \mathrm{S}, 14.35$.

\section{Pharmacology}

Swiss albino mice weighing 30-35g were used in the present study. Laboratory temperature was maintained at $20 \pm 1{ }^{\circ} \mathrm{C}$ under conditions of a 12 hour light dark schedule. Before experimentation, mice were allowed 1 week of adaptation. They were used only once. The study was approved by the Local Ethics Committee of Eskisehir Osmangazi University for The Care and Use of Laboratory Animals. The animals were divided into 25 groups. Each group included seven animals. All compounds were dissolved in DMSO / water (1:4) and given to the animals per orally at $100 \mathrm{mg} / \mathrm{kg}$ doses. Control animals received orally $0.1 \mathrm{~mL}$ DMSO / water (1:4). Tail clip test, tail flick test to radiant heat, hot plate test, and writhing test induced by acetic acid were performed 60 minutes after the administration of the compounds or vehicle (DMSO for control group).

\section{Tail clip test}

This analgesic test is based on a method as described by Bianchi and Franceschini (1954), and Dajani et al. (1999). A pressure-standardized artery clip was placed approximately $2 \mathrm{~cm}$ from the base of tail and only the mice that responded to the clip placement by turning or biting the clip within 15 seconds were used in this test.

\section{Tail flick test to radiant heat}

This test described by D'Amour and Smith was done with a beam of high-intensity light focused on the dorsal surface of the tail (D'Amour, Smith, 1941). The response latency between the onset of the radiant heat stimulus and the movement of the tail out of the light beam of the apparatus (MAY, produced in Turkey) was determined. The light intensity was set to provide a predrug response time of 2-4 seconds. A 15 second cut off was used in order to prevent damage to the tail.

\section{Hot plate test}

The test was based on the description by Eddy and Leimbach (1953) and Noble, Smadja and Roques (1994). A glass cylinder (16 mm high $16 \mathrm{~mm}$ diameter) was used to keep the mouse on the heated surface of the plate which was kept at a temperature of $55 \pm 0.5^{\circ} \mathrm{C}$ by using a thermoregulated water circulating pump. The latency period until the mouse licked a foot or jumped was registered by a means of a stopwatch (cutoff time $45 \mathrm{~s}$ ). The results were expressed as the percent of the maximal possible effect $(\% \mathrm{MPE} \pm \mathrm{SD})$.

$$
\% \mathrm{MPE}=\frac{\text { postdrug latency }- \text { predrug latency }}{\text { cutoff time }- \text { predrug latency }} \times 100
$$

\section{Writhing test}

Abdominal constrictor test was performed by the i.p. application of $0.6 \%$ acetic acid $(60 \mathrm{mg} / \mathrm{kg})$ and stretching movements (arching of the back, development of tension in the abdominal muscles, elongation of the body and extension of forelimbs) were counted in a period of 10 min starting 5 min after the i.p. administration of acetic acid (Koster, Anderson, De Beer, 1959). All tests were conducted between 9 and 12 a.m.

All results were expressed as mean of \pm S.D. Statistical comparison was performed by using Student's $\mathrm{t}$ test.

\section{Cyclooxygenase inhibitory assay}

Inhibitory effects of the test compounds on COX1 and COX-2 activities were investigated by using Cayman's Colorimetric COX (ovine) Inhibitor Screening Assay Kit (Cayman Chemical, Ann Arbor, MI, USA, Catalog No:760111; Lot Number: 043345). DMSO was used as solvent control. SC-560 for COX-1 and DuP697 for COX-2 were used as reference standards. Each compound was tested at $10 \mu \mathrm{M}$ and all experiments were performed in triplicate.

\section{RESULTS AND DISCUSSION}

\section{Chemistry}

The synthesis of 3-[(6-substituted phenyl[1,2,4] triazolo[3,4- $b][1,3,4]$ thiadiazol-3-yl)methyl]-2(3H)benzoxazolone (5a-n) and 3-[(6-substitutedbenzyl[1,2,4] triazolo[3,4-b][1,3,4] thiadiazol-3-yl)methyl]-2(3H)benzoxazolone (6a-h) were accomplished as presented in Figure 1. Synthesis of 2(3H)-benzoxazolone 1 (Eren et al. $2010)$, ethyl-(2(3H)-benzoxazolone-3-yl)acetate 2 (Onkol et al. 2002) , (2(3H)-benzoxazolone-3-yl)acetic acid 3 (Onkol et al. 2002) and 3-[(4-amino-5-thioxo1,2,4-triazol-3-yl)methyl]-2(3H)-benzoxazolone 4 (Urlu Cicekli et al. 2012) was accomplished according to the previously reported procedures. 3-[(4-Amino-5-thioxo1,2,4-triazol-3-yl)methyl]-2(3H)-benzoxazolone 4 was treated with substituted benzoic acid or phenylacetic acids using $\mathrm{POCl}_{3}$ as cyclizing agent under microwave irradiation 
to yield 3-[(6-substitutedphenyl[1,2,4]triazolo[3,4-b] $[1,3,4]$ thiadiazol-3-yl)methyl]-2(3H)-benzoxazolone (5a-n) and 3-[(6-substitutedbenzyl[1,2,4]triazolo[3,4-b] [1,3,4]thiadiazol-3-yl)methyl]-2(3H)-benzoxazolone (6ah) in good yields (Figure 1). Advantages of microwave irradiation such as high yield, short reaction time, pure product, and easy work up prompted us to synthesize compounds under microwave irradiation.

The structures of the synthesized compounds were elucidated by IR, ${ }^{1} \mathrm{H}-\mathrm{NMR},{ }^{13} \mathrm{C}-\mathrm{NMR}$, LC-MS, and elemental analysis.

IR spectroscopic data of the compound $\mathbf{4}$ structure showed two characteristic absorption bonds, one of which appearing at $2585 \mathrm{~cm}^{-1}$ was attributed to $\mathrm{SH}$ and the other at $3200-3300 \mathrm{~cm}^{-1}$ was assigned to $\mathrm{NH}_{2}$. However, both bonds disappeared with the formation of triazolothiadiazole derivatives (5a-n, 6a-h).

In the ${ }^{1} \mathrm{H}-\mathrm{NMR}$ spectrum of $\mathbf{5 a - n}, \mathbf{6 a - h}$, a singlet that appeared at $\delta 5.60 \mathrm{ppm}$ was attributed to the methylene protons and $\mathbf{6 a}-\mathbf{h}$ exhibited the $-\mathrm{CH}_{2}-\mathrm{C}_{6} \mathrm{H}_{5}$ signals as a singlet between $\delta 4.33$ and $4.58 \mathrm{ppm}$.

\section{Biological activity}

The antinociceptive activity of the all compounds were evaluated using both chemical and thermal methods of nociception. These methods are used to detect the central and peripheral mechanisms of analgesia. Acetic acid induced writhing test is used for detecting peripheral analgesia, whereas tail flick test, hot plate and tail clip test are sensitive to centrally acting analgesia. In order to have a standard drug for comparison, the compounds were tested at an equimolar oral dose relative to $100 \mathrm{mg} / \mathrm{kg}$ aspirin. The percentage inhibition was calculated after 60 min. The effect of synthesized compounds is summarized in Table I.

The tail clip test revealed that the compounds $\mathbf{5 i}$ and $\mathbf{6 e}$ exhibited moderate antinociceptive activity in comparison to the standard drug aspirin.

Compounds $\mathbf{5 f}, \mathbf{5 g}, \mathbf{5 m}$ and $\mathbf{6 c}$ were more effective than aspirin in the tail flick test. It was observed that triazolothiadiazole derivatives having 4-terbutylphenyl (5f), 4-methylphenyl (5g), 3-thienyl (5m)

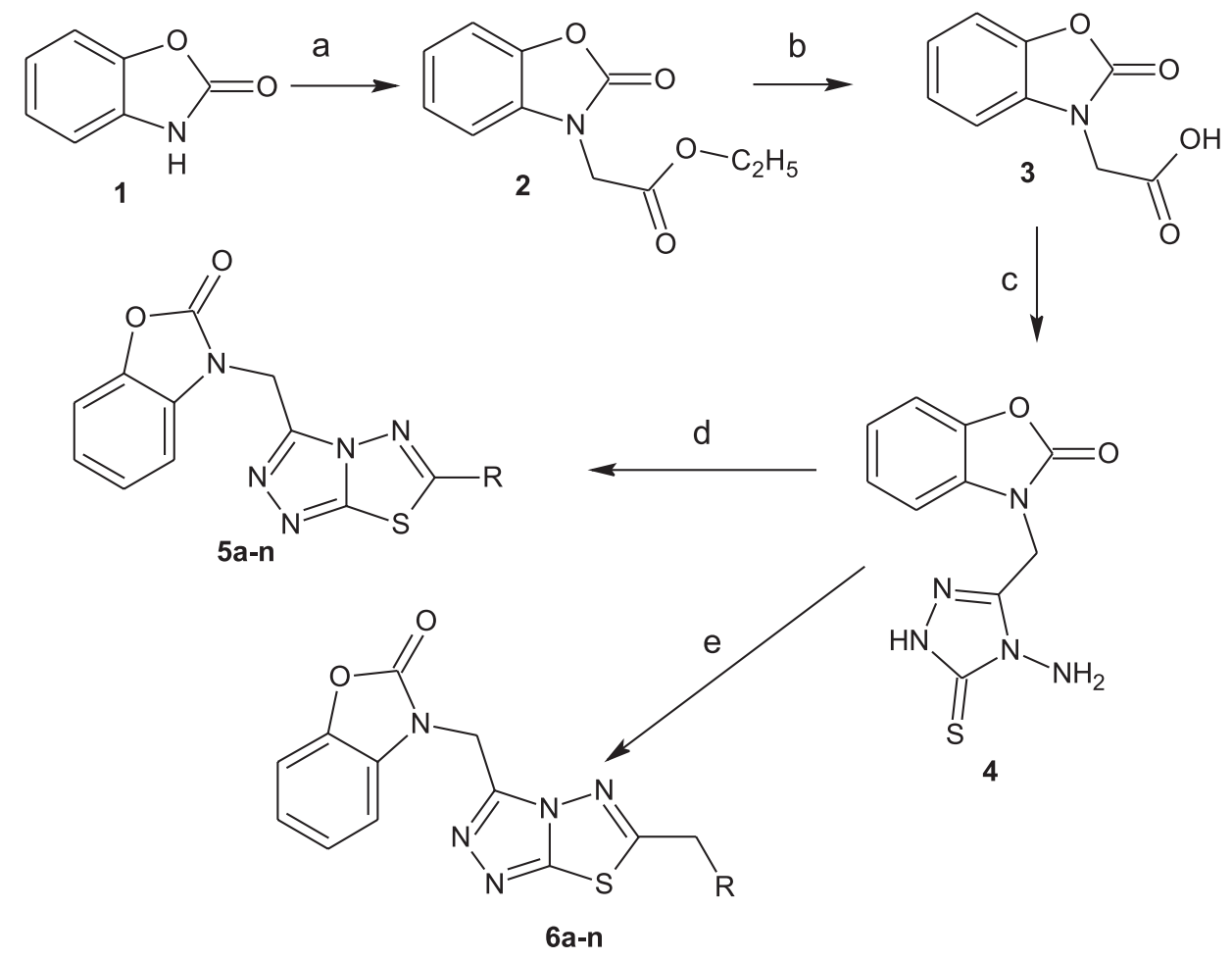

a: $\mathrm{BrCH}_{2} \mathrm{COOC}_{2} \mathrm{H}_{5} /$ Aceton $/ \mathrm{K}_{2} \mathrm{CO}_{3} / \mathrm{MW}$ b: $\mathrm{HCl}$

$$
\begin{aligned}
& \text { c: } \mathrm{NH}_{2} \mathrm{NHCSNHNH}_{2} \quad \text { d: } \mathrm{C}_{6} \mathrm{H}_{5} \mathrm{COOH} / \mathrm{POCl}_{3} / \mathrm{MW} \quad \text { e: } \mathrm{C}_{6} \mathrm{H}_{5} \mathrm{CH}_{2} \mathrm{COOH} / \mathrm{POCl}_{3} / \mathrm{MW} \\
& \mathrm{R}(5 \mathrm{a}-\mathrm{n}): \mathrm{H}, \mathrm{Cl}, \mathrm{F}, \mathrm{Br}, \mathrm{OCH}_{3}, \mathrm{Br}, \mathrm{CH}_{3}, \mathrm{CN}, \mathrm{C}\left(\mathrm{CH}_{3}\right)_{3}, \mathrm{SO}_{2} \mathrm{CH}_{3}, \mathrm{NO}_{2} \\
& \mathrm{R}(6 \mathrm{a}-\mathrm{h}): \mathrm{H}, \mathrm{F}, \mathrm{Cl}, \mathrm{CH}_{3}, \mathrm{OCH}_{3}, \mathrm{CF}_{3}, \mathrm{C}\left(\mathrm{CH}_{3}\right)_{3}, \mathrm{SO}_{2} \mathrm{CH}_{3}
\end{aligned}
$$

FIGURE 1 - Synthesis of compounds 5a-n and 6a-h. 
TABLE I - Antinociceptive activity of synthesised compounds

\begin{tabular}{|c|c|c|c|c|c|}
\hline Comp. & $\mathbf{R}$ & $\begin{array}{l}\text { Tail Clip } \\
\text { \% MPE }\end{array}$ & $\begin{array}{l}\text { Tail Flick } \\
\% \text { MPE }\end{array}$ & $\begin{array}{l}\text { Hot Plate } \\
\% \text { MPE }\end{array}$ & $\begin{array}{c}\text { Writting test } \\
\text { Stretching number }\end{array}$ \\
\hline Control & & $4,11 \pm 2,72$ & 0 & $12,41 \pm 5,09$ & $37,80 \pm 10,03$ \\
\hline 4 & & $32,61 \pm 19,66^{*}$ & $64,25 \pm 19,14 *$ & $2,14 \pm 1,37$ & $19,33 \pm 9,10^{*}$ \\
\hline $5 \mathrm{a}$ & phenyl & $38,03 \pm 13,64^{*}$ & $45,11 \pm 18,46^{*}$ & $10,75 \pm 6,91$ & $33,83 \pm 9,80$ \\
\hline $5 b$ & 4-fluorophenyl & $33,05 \pm 15,54^{*}$ & $16,85 \pm 6,79^{*}$ & $6,25 \pm 4,06$ & $8,50 \pm 6,59^{*+}$ \\
\hline $5 c$ & 4-chlorophenyl & $35,68 \pm 15,93^{*}$ & $33,33 \pm 21,08^{*}$ & $31,85 \pm 14,68^{*+}$ & $20,33 \pm 7,36^{*}$ \\
\hline $5 d$ & 4-bromophenyl & $3,16 \pm 1,77$ & $46,08 \pm 20,89^{*}$ & $17,50 \pm 6,86$ & $21,83 \pm 6,82 *$ \\
\hline $5 e$ & 4-methoxyphenyl & $28,28 \pm 14,68^{*}$ & $34,25 \pm 14,58^{*}$ & $5,42 \pm 2,87$ & $22,17 \pm 7,61^{*}$ \\
\hline $5 \mathrm{f}$ & 4-tert-butylphenyl & $17,56 \pm 12,43^{*}$ & $77,69 \pm 14,13^{*+}$ & $15,12 \pm 8,59$ & $13,83 \pm 4,62^{*+}$ \\
\hline $5 \mathrm{~g}$ & 4-methylphenyl & $36,12 \pm 20,37^{*}$ & $66,67 \pm 21,08^{*+}$ & $24,55 \pm 15,40$ & $7,00 \pm 3,07^{*+}$ \\
\hline $5 \mathrm{~h}$ & 4-(trifluoromethyl)phenyl & $3,98 \pm 2,40$ & $19,00 \pm 16,36^{*}$ & $3,84 \pm 1,31$ & $43,20 \pm 8,76$ \\
\hline $5 \mathrm{i}$ & 4-(methylsulfonyl)phenyl & $40,77 \pm 13,79^{*}$ & $58,50 \pm 19,22^{*}$ & $24,77 \pm 13,32$ & $13,50 \pm 7,67^{*}$ \\
\hline $5 \mathrm{j}$ & 4-nitrophenyl & $26,30 \pm 16,79 *$ & $54,17 \pm 20,83^{*}$ & $8,17 \pm 5,07$ & $6,17 \pm 2,91^{*+}$ \\
\hline $5 \mathrm{k}$ & 4-cyanophenyl & $23,33 \pm 16,66^{*}$ & $26,15 \pm 17,25^{*}$ & 0 & $30,67 \pm 10,90$ \\
\hline 51 & pyridin-4-yl & $7,25 \pm 4,41$ & $36,00 \pm 17,29^{*}$ & $18,45 \pm 8,56$ & $11,83 \pm 4,31^{*+}$ \\
\hline $5 \mathrm{~m}$ & thiophen-3-yl & $9,21 \pm 6,44$ & $68,83 \pm 19,78^{*+}$ & $4,83 \pm 2,81$ & $31,17 \pm 4,85$ \\
\hline $5 n$ & furan-3-yl & $14,34 \pm 7,70^{*}$ & $45,32 \pm 16,11^{*}$ & $10,93 \pm 5,52$ & $6,33 \pm 2,32^{*+}$ \\
\hline $6 a$ & phenyl & 0 & 0 & $14,83 \pm 5,42$ & $27,50 \pm 9,28$ \\
\hline $6 b$ & 4-fluorophenyl & $33,33 \pm 21,05^{*}$ & $44,25 \pm 19,64^{*}$ & 0 & $10,00 \pm 5,03^{*+}$ \\
\hline $6 c$ & 4-chlorophenyl & $37,65 \pm 17,33^{*}$ & $63,94 \pm 17,29^{*+}$ & $20,96 \pm 13,59$ & $25,33 \pm 7,88^{*}$ \\
\hline $6 \mathrm{~d}$ & 4-methylphenyl & $16,67 \pm 6,80^{*}$ & $29,73 \pm 19,01^{*}$ & $18,07 \pm 12,28$ & $6,50 \pm 4,23^{*+}$ \\
\hline $6 e$ & 4-methoxyphenyl & $41,26 \pm 20,04 *$ & 0 & $9,26 \pm 3,29$ & $22,50 \pm 5,96^{*}$ \\
\hline $6 f$ & 4-(trifluoromethyl)phenyl & $5,77 \pm 3,89^{*}$ & $21,56 \pm 15,85^{*}$ & $6,46 \pm 3,34$ & $14,83 \pm 6,12^{*}$ \\
\hline $6 \mathrm{~g}$ & 4-tert-butylphenyl & $19,34 \pm 13,09^{*}$ & $38,35 \pm 15,47^{*}$ & $1,55 \pm 1,21$ & $23,17 \pm 6,98^{*}$ \\
\hline $6 \mathrm{~h}$ & 4-(methylsulfonyl)phenyl & $33,46 \pm 16,34^{*}$ & $47,27 \pm 21,28^{*}$ & $4,29 \pm 2,04$ & $24,67 \pm 6,45^{*}$ \\
\hline Aspirin & & $43,67 \pm 20,49 *$ & $37,39 \pm 20,10 *$ & $13,36 \pm 6,57$ & $22,50 \pm 7,97^{*}$ \\
\hline
\end{tabular}

(MPE : Maximum possible effect; all the values are given as $\mathrm{X} \pm \mathrm{SD} ; \mathrm{n}: 7) ; * P<0.05$; as compared to control; $+: P<0.05$; as compared to aspirin.

and 4-chlorophenyl (6c) groups showed good activity with $77.69 \%, 66.67 \%, 68.83 \%$ and $63.94 \%$, respectively.

The hot plate test is generally used for centrally acting analgesic drugs. In this study, compound 5c (4-chlorophenyl) was more effective than aspirin and compound $\mathbf{5 g}$ (4-methylphenyl), 5i (4-methylsulfonylphenyl), and $\mathbf{6 c}$ (4-chlorophenyl) were as effective as aspirin. Additionally, compound 5c (4-chlorophenyl), 5g (4-methylphenyl), 5i (4-methylsulfonylphenyl) and 6c (4-chlorophenyl) were more effective than compound 4 in the hot plate test. It was observed that triazolothiadiazole derivatives having 4-chlorophenyl (5c), 4-tert-butylphenyl (5f), 4-methylsulfonylphenyl (5i), 4-chlorophenyl (6c), and 4-methylphenyl (6d) groups also showed good activity.

The acetic acid-induced writhing method was widely used for the evaluation of peripheral antinociceptive activity. This method is considered to be a non-selective antinociceptive model. Compound $\mathbf{5 b}$ (4-fluorophenyl) , $\mathbf{5 f}$ (4-tert-butylphenyl), 5g (4-methylphenyl), $\mathbf{5 j}$ (4-nitrophenyl), 5l (pyridin-4-yl), 5n (furan-3-yl), $6 \mathbf{b}$ (4-fluorobenzyl), and 6d (4-methylphenyl) exhibited higher antinociceptive activity in writhing test when compared with aspirin and compound 4.

The effects of compounds on peripheral nervous system were found to be higher than central nervous system. Therefore, few compounds exhibiting significant in vivo antinociceptive activity were screened for their in vitro COX-1 and COX-2 inhibitory activity (Figure 2) by using colorimertic $\mathrm{COX}$ inhibitor screening assay method. SC-560 for COX-1 and DuP-697 for COX-2 were used 


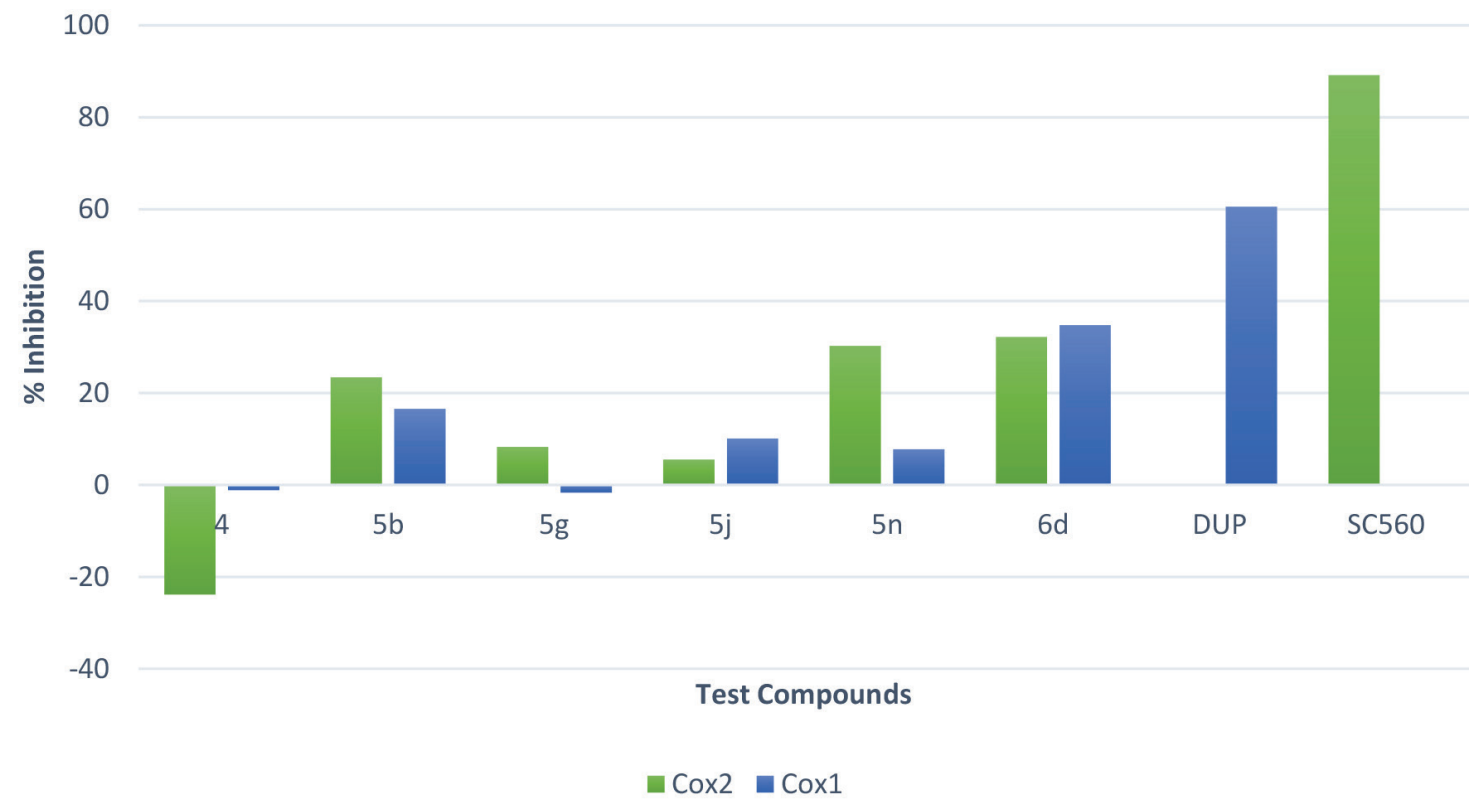

FIGURE 2 - COX-1 and COX-2 Inhibitory activities of test compounds at $10 \mu \mathrm{M}$ concentration.

as reference standards. Except for compound 6d, the test compounds showed no significant difference compared to reference standards. The percentage inhibition of in-vitro COX inhibition was depicted in Figure 2. Compound 6d showed the highest COX-1 and COX-2 inhibition rate with $34.79 \%$ and $32.19 \%$, respectively.

Compound 5i showed significant activity in the tests which indicates that this compound shows its activity peripherally as well as centrally. Substitution of heteroaromatic groups on compound $\mathbf{4}$ decreases the antinociceptive activity which is comparable with the reference drugs. Also, the effect of electron withdrawing groups has increased in hot plate, tail flick and tail click tests. On the other hand, compound $\mathbf{5 g}$ could be centrally active whereas compound $\mathbf{5} \mathbf{j}$ might be peripherally active.

I t w a s a $1 \mathrm{so}$ not i ced that $\mathrm{the}$ 3 -[(6-substituedphenyl[1,2,4] triazolo[3,4-b][1,3,4] thiadiazol-3-yl)methyl]-2(3H)-benzoxazolone derivatives (5a-n) showed higher activity than 3 -[(6-substituebenzyl[1,2,4]triazolo[3,4-b][1,3,4] thiadiazol-3-yl)methyl]-2(3H)-benzoxazolone derivatives (6a-h) in this study. These results suggest that these compounds could possibly have peripheral antinociceptive activity.

\section{ETHICS STATEMENT}

The experimental protocols were approved in accordance with the Guide for the Care and Use of Laboratory Animals at Eskisehir Osmangazi University (Protocol number; 31.03.2011/200).

\section{CONCLUSION}

In this study, new series of 3 -substituted $[1,2,4]$ triazolo[3,4-b][1,3,4]thiadiazol-3-yl)methyl]-2(3H)benzoxazolone derivatives were synthesized and their antinociceptive activity was determined. Among these compounds, compound $\mathbf{5 g}$ and $\mathbf{5 j}$ have been found to be significantly more active than the other compounds and the standard in the tests. The mechanism of the biological activity needs further investigations.

\section{ACKNOWLEDGMENTS}

This study was supported financially with a grant from the Research Foundation of Gazi University ( Project No: 02/2009-03 and 02/2010-22).

\section{REFERENCES}

Abdelazeem AH, Khan SI, White SW, Sufka KJ, McCurdy CR. Design, synthesis and biological evaluation of bivalent benzoxazolone and benzothiazolone ligands as potential anti-inflammatory/analgesic agents. Bioorganic Med Chem. 2015;23(13):3248-3259.

Aggarwal KJM, Kumar N, Dureja P. Synthesis, antimicrobial evaluation and QSAR analysis of novel nalidixic acid based 1,2,4-triazole derivatives. Eur J Med Chem. 2011;46(9):40894099 . 
Aichaoui H, Guenadil F, Kapanda CN, Lambert DM, McCurdy CR, Poupaert JH. Synthesis and pharmacological evaluation of antioxidant chalcone derivatives of $2(3 \mathrm{H})$-benzoxazolones. Med Chem Res. 2009;18(6):467-476.

Akhter MW, Hassan MZ, Amir M. Synthesis and pharmacological evaluation of 3-diphenylmethyl-6-substituted-1,2,4-triazolo[3,4b]-1,3,4-thiadiazoles: A condensed bridgehead nitrogen heterocyclic system. Arab J Chem. 2014;7(6):955-963.

Bianchi J, Franceschini C. Experimental observations on haffner's method for testing analgesic drugs. Br J Pharmacol Chemother. 1954;9(3):280-284.

D’Amour DL, Smith FE. A method for determinatin loss of pain sensation. J Pharmacol Exp Ther. 1941;72(1):74-79.

Dajani EZ, Larsen KR, Taylor J, Dajani NE, Shahwan TG, Neeleman SD, Taylor MS, Dayton MT, Mir GN. 1',1'-Dimethylheptyl-delta-8-tetrahydrocannabinol-11-oic acid: a novel, orally effective cannabinoid with analgesic and anti-inflammatory properties. J Pharmacol Exp Ther. 1999;291(1):31-38.

Dannhardt G, Kiefer W. Cyclooxygenase inhibitors--current status and future prospects. Eur J Med Chem. 2001;36(2):109126.

Dogruer DS, Unlu S, Yesilada E, Sahin MF. N-(2-pyridinyl)-2[2(3H)-benzazolone-3-yl]acetamides: Synthesis, antinociceptive and anti-inflammatory activity. Farmaco. 1997;52(12):745-750.

Eddy D, Leimbach NB. Synthetic analgesics. II. Dithienylbutenyl- and dithienylbutylamines. J Pharmacol Exp Ther. 1953;107(3):385-393.

Eren G, Ünlü S, Nuñez MT, Labeaga L, Ledo F, Entrena A, Banoglu E, Costantino G, Sahin MF. Synthesis, biological evaluation, and docking studies of novel heterocyclic diaryl compounds as selective COX-2 inhibitors. Bioorganic Med Chem. 2010;18(17):6367-6376.

Gabra P, Sirois BH. Beneficial effect of chronic treatment with the selective bradykinin B1 receptor antagonists, R-715 and R-954, in attenuating streptozotocin-diabetic thermal hyperalgesia in mice. Peptides. 2003;24(8):1131-1139.

Gokhan-Kelekci N, Koksal M, Unuvar S. Synthesis and characterization of some new $2(3 \mathrm{H})$ - benzoxazolones with analgesic and antiinflammatory activities. J Enzym Inhib Med Chem. 2009;24(1):29-37.
Gulkok Y, Biçer T, Onurdağ FK, Ozgen S, Şahin MF, Doğruer DS. Synthesis of some new urea and thiourea derivatives and evaluation of their antimicrobial activities. Turkish J Chem. 2012;36:279-291.

Haider S, Alam MS, Hamid H, Dhulap A, Umar S, Yar MS, Bano S, Nazreen S, Ali Y, Kharbanda C. Design, synthesis and docking studies of 2-benzoxazolinone-based 1,2,4-triazoles as proinflammatory cytokine inhibitors. Med Chem Res. 2014;23(9):4250-4268.

Hussein MA, Shaker RM, Ameen MA, Mohammed MF. Synthesis, anti-inflammatory, analgesic, and antibacterial activities of some triazole, triazolothiadiazole, and triazolothiadiazine derivatives. Arch Pharm Res. 2011;34(8):1239-1250.

Ibrahim DA. Synthesis and biological evaluation of 3,6-disubstituted $[1,2,4]$ triazolo[3,4-b][1,3,4]thiadiazole derivatives as a novel class of potential anti-tumor agents. Eur J Med Chem. 2009;44(7):2776-2781.

Koksal M, Gokhan N, Erdogan H, Ozalp M, Ekizoglu M. Synthesis of 3- (4-substituted benzoylmethyl) -2-benzoxazolinones and screening antimicrobial activities. Farmaco. 2002;57:535-538.

Koster J, Anderson R, De Beer M. Acetic acid for analgesic screening. Fed Proc. 1959;18:412-417.

Kritsanida M, Mouroutsou A, Marakos P, Pouli N, Papakonstantinou-Garoufalias S, Pannecouque C, Witvrouw M, De Clercq E. Synthesis and antiviral activity evaluation of some new 6-substituted 3-(1-adamantyl)-1,2,4-triazolo[3,4-b] $[1,3,4]$ thiadiazoles. Farmaco. 2002;57(3):253-257.

Kumar GVS, Rajendraprasad Y, Mallikarjuna BP, Chandrashekar SM, Kistayya C. Synthesis of some novel 2-substituted-5[isopropylthiazole] clubbed 1,2,4-triazole and 1,3,4-oxadiazoles as potential antimicrobial and antitubercular agents. Eur J Med Chem. 2010a;45(5):2063-2074.

Kumar GVS, Rajendraprasad Y, Mallikarjuna BP, Chandrashekar SM. Synthesis and pharmacological evaluation of clubbed isopropylthiazole derived triazolothiadiazoles, triazolothiadiazines and mannich bases as potential antimicrobial and antitubercular agents. Eur J Med Chem. 2010b;45(11):5120-5129.

Mathew V, Keshavayya J, Vaidya VP, Giles D. Studies on synthesis and pharmacological activities of 3,6-disubstituted1,2,4-triazolo[3,4-b]-1,3,4-thiadiazoles and their dihydro analogues. Eur J Med Chem. 2007;42(6):823-840. 
Mathew V, Keshavayya J, Vaidya VP. Heterocyclic system containing bridgehead nitrogen atom: synthesis and pharmacological activities of some substituted 1,2,4-triazolo[3,4b]-1,3,4-thiadiazoles. Eur J Med Chem. 2006;41(9):1048-1058.

Noble C, Smadja BP, Roques F. Role of endogenous cholecystokinin in the facilitation of $M u$-mediated antinociception by Delta-opioid agonists. J Pharmacol Exp Ther. 1994;271(3):1127-1134.

Onkol T, Dundar Y, Sirmagul B, Erol K, Sahin MF. (2-Oxobenzazolin-3-yl)alkanoic acid derivatives and antinociceptive activity. J Fac Pharm Gazi. 2002;19(1):15-24.

Petrov O, Ivanova Y, Momekov G, Kalcheva V. New synthetic chalcones: Cytotoxic Mannich bases of 6-(4-chlorocinnamoyl)2(3H)-benzoxazolone. Lett Drug Design Discov. 2008;5(6):358361.

Sarigol D, Uzgoren-Baran A, Tel BC, Somuncuoglu EI, Kazkayasi I, Ozadali-Sari K, Unsal-Tan O, Okay G, Ertan M, Tozkoparan B. Novel thiazolo[3,2-b]-1,2,4-triazoles derived from naproxen with analgesic/anti-inflammatory properties: Synthesis, biological evaluation and molecular modeling studies. Bioorg Med Chem. 2015;23(10):2518-2528.

Satyendra RV, Vishnumurthy KA, Vagdevi HM, Rajesh KP, Manjunatha H, Shruthi A. Synthesis, in vitro antioxidant, anthelmintic and molecular docking studies of novel dichloro substituted benzoxazole-triazolo-thione derivatives. Eur J Med Chem. 2011;46(7):3078-3084.
Sharma M, Deekshith V, Semwal A. Discovery of fused Triazolothiadiazoles as Inhibitors of TNF-alpha: pharmacophore hybridization for treatment of neuropathic pain. Pain Ther. 2012;1(1):1-17.

Shiradkar MR, Akula KC, Dasari V, Baru V, Chiningiri B, Gandhi S, Kaur R. Clubbed thiazoles by MAOS: A novel approach to cyclin-dependent kinase 5/p25 inhibitors as a potential treatment for Alzheimer's disease. Bioorg Med Chem. 2007;15(7):2601-2610.

Ucar H, Derpoorten KV, Spampinato S, Stables JP, Depovere P, Masereel B, Delarge J. Synthesis and anticonvulsant activity of $2(3 \mathrm{H})$ - benzoxazolone and 2(3H)-benzothiazolone derivatives. J Med Chem. 1998;41(7):1138-1145.

Unlu S, Onkol T, Dundar Y, Okçelik B, Küpeli E, Yeşilada E, Noyanalpan N, Şahin MF. Synthesis and analgesic and antiinflammatory activity of some new (6-acyl-2-benzoxazolinone and 6-acyl-2-benzothiazolinone derivatives with acetic acid and propanoic acid residues. Arch Pharm. 2003;336(8):353-360.

Urlu Cicekli S, Onkol T, Ozgen S, Sahin MF. Schiff bases of 3-[(4-amino-5-thioxo-1,2,4-triazole-3-yl) methyl]-2(3H)benzoxazolone derivatives: Synthesis and biological activity. Rev Roum Chim. 2012;57(3):187-195.

Wong CH, Dey P, Yarmush J, Wu WH, Zbuzek VK. Nifedipineinduced analgesia after epidural injection in rats. Anesth Analg. 1994;79(2):303-306.

Received for publication on $21^{\text {st }}$ February 2018 Accepted for publication on $23^{\text {rd }}$ October 2018 